\title{
Rigenerazione urbana e public history: il progetto Atr Contemporaneo di Forlì
}

L'articolo parte da un progetto di stage per il Master di II livello in Public History di Unimore, condotto con l'Associazione culturale Città di Ebla e Spazi indecisi. La restituzione al pubblico dell'edificio dell'ex deposito Atr di Forli in occasione del festival culturale Ipercorpo è stata affrontata con gli strumenti della public history, offrendo l'occasione per una ricerca d'archivio e una riflessione sull'apporto che le architetture razionaliste in disuso possono fornire alla città.

The article starts from a master's degree project in Public History of Unimore, conducted with the Cultural Association Città di Ebla and Spazi indecisi. The renovation of the former Atr Depot in Forli on the occasion of the Ipercorpo Cultural Festival was dealt with the tools of public history, giving the opportunity for an archival research and a reflection on the contribution that disused rationalist architectures can provide for the city.

\section{Il progetto Atr Contemporaneo e i suoi promotori: Città di Ebla e Spazi indecisi}

La Romagna e la città di Forlì hanno rappresentato un laboratorio fondamentale per il regime fascista nella costruzione del consenso. Essenziali in questo processo sono state le costruzioni pubbliche messe in piedi sia per favorire la modernizzazione della società, sia per creare luoghi di aggregazione utili al consenso. Forlì presenta monumenti ed edifici simbolo di questa particolare politica i quali possono essere "letti" in vari modi. Uno di questi è quello di focalizzare l'attenzione sulla vita quotidiana legata a questi punti della città. [Troilo 2014] 
Con queste parole, Matteo Troilo coglie nel segno nell'evidenziare l'importanza che in epoca fascista Forlì rivestì per l'allestimento di un'architettura di regime. Oggi questa ingombrante eredità è oggetto di vari progetti di rigenerazione urbana, tra i quali il progetto Atr Contemporaneo volto al recupero di un edificio in sta-

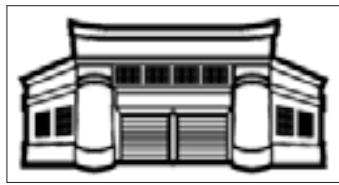

Fig. 1. Logo del progetto Atr Contemporaneo. to di abbandono come quello dell'ex stabilimento Sita (Società italiana trasporti automobilistici).

I partner principali di questa operazione sono l'associazione culturale Città di Ebla e il collettivo Spazi Indecisi. La prima è nata a Forlì nel 2004 sotto la guida di Claudio Angelini. Da subito radicata nell'ambiente cittadino, dal 2006 promuove il festival Ipercorpo, che si afferma presto come un importante punto di incontro del panorama artistico giovanile internazionale, pur ponendosi come una realtà in fieri, volutamente in evoluzione, per richiamare la liquidità e l'incertezza del presente. Il secondo scaturisce nel 2009 dall'incontro di diversi giovani professionisti, uniti dalla volontà di fare rivivere spazi urbani inutilizzati, abbandonati, ormai privi di funzione - in questo senso, "indecisi" -, al fine di dar loro una nuova valorizzazione attraverso l'utilizzo per attività di aggregazione culturale. Non viene cancellata la loro storia, anzi: la modalità del riuso leggero riporta a galla presso la comunità locale il lascito di storie che ogni luogo porta con sé. Questa ridefinizione dei luoghi abbandonati trasforma l'abbandono in risorsa, il degrado consumista viene utilizzato come opportunità di abbracciare il cambiamento, innescando processi di rigenerazione urbana leggera.

Nel 2009 nasce il legame tra Città di Ebla e Atr (Azienda trasporti romagnoli) Agenzia per la mobilità della Provincia di Forlì-Cesena [Nicosanti 2015, 124], che frutterà alla piattaforma di Ipercorpo la possibilità di organizzare le nuove edizioni del festival in uno spazio unico: il complesso Sita, poi Atr, deposito abbandonato delle corriere sito in Largo Savonarola a Forlì, in centro storico.

Nel 2013 Città di Ebla, insieme a Spazi indecisi (come co-gestori), ad Atr e al Comune di Forlì (capofila del progetto) partecipa alla manifestazione d'interesse per il programma 5.1 della Regione Emilia-Romagna "Sostegno allo sviluppo delle infrastrutture per la competitività del territorio" (Delibera regionale n.1112 del 2 agosto 2013) per il triennio 2012-2015. Il progetto proposto, come descrive l'organizzatrice e amministratrice di Città di Ebla Elisa Nicosanti,

desidera favorire la convivenza di incubatori d'impresa e sperimentazione artistica-culturale in un unico luogo di confronto e partecipazione per una contaminazione reciproca, dove le attività di professionisti e giovani che operano nel mondo della creatività e dell'innovazione convivano con l'arte performativa contemporanea, rigenerando e riattivando un luogo di grande valore architettonico come l'ex 
deposito Atr. [Nicosanti 2015, 131]

La proposta avanzata da Città di Ebla e Spazi indecisi, coadiuvati da Comune e Atr, viene giudicata valida e la giunta della Regione Emilia-Romagna concede la gestione triennale degli spazi dell'ex stabilimento Sita. Il cambio della giunta comunale e regionale e alcune reticenze dell'amministrazione locale hanno però imposto uno slittamento dei tempi di realizzazione del progetto, che è partito fattivamente alla fine del 2015 e che si focalizzerà sul triennio 2016-2018.

L'obiettivo proposto è riattivare l'ex deposito Atr mutandone la destinazione d'uso, rendendolo un luogo di connessione tra mondo artistico e mondo dell'impresa, per favorire l'innovazione culturale e sociale. Per la sua realizzazione, oltre a interventi strutturali sull'immobile, il progetto si articola in una serie di attività e prevede il potenziamento e la messa in relazione della progettualità delle due associazioni, appunto Città di Ebla e Spazi indecisi. Questo viene già sperimentato tra il 2011 e il 2014, quando le due associazioni promuovono il festival Ipercorpo, l'evento Cicli Indecisi e 1'esposizione Totally Lost all'interno degli spazi dell'ex deposito. Viene così testato il potenziale degli spazi dell'ex Sita, che si dimostrano versatili e di grande effetto, ma che necessitano di alcuni interventi strutturali.

Fig. 2.

Festival

Ipercorpo

all'ex

deposito

Atr (foto di

Gianluca

Campore-

si).

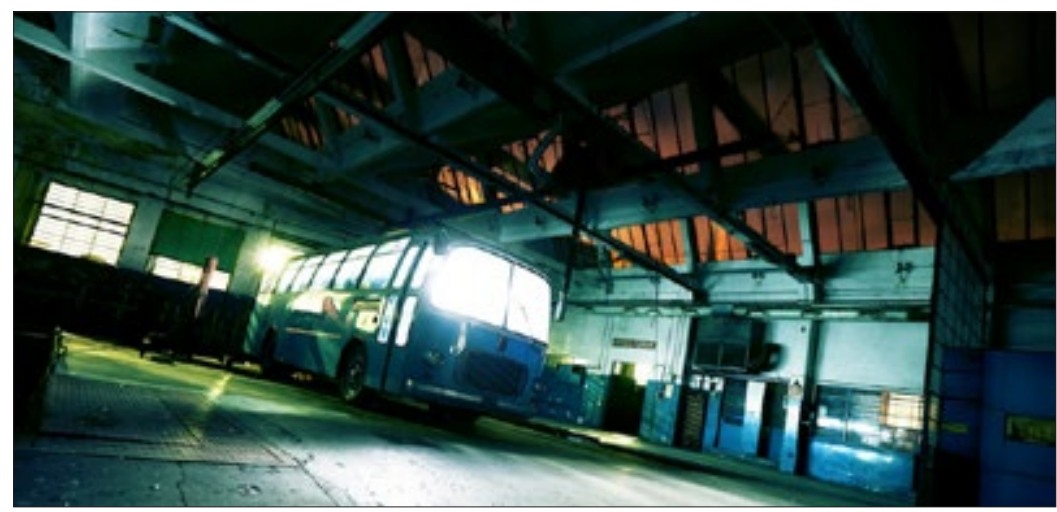

Le edizioni 2015 e 2016 di Ipercorpo non si sono potute svolgere nell'ex deposito Atr proprio a causa delle condizioni dell'edificio, che non erano ancora atte a ospitare un evento che raccoglie un seguito sempre più numeroso. Si è dunque scelto di disseminare il festival in più luoghi, di pari passo con la diversificazione sempre più accentuata dell'offerta artistica, articolata tra teatro, musica, arte, danza. L'edizione 2016 ha avuto come centro nevralgico un altro luogo 'indeciso', l'ex Centrale avicola Amadori, nella prima periferia forlivese.

Durante la conferenza stampa di presentazione del progetto al pubblico, il 30 giugno 2016, Claudio Angelini ha parlato di ciò che diventerà 1'ex deposito delle 
corriere come di «un hub, cioè uno spazio aperto e di connessione dove imprese culturali e creative possano fare da cerniera fra imprese di lavoro cittadine, arte, creatività e territorio, producendo innovazione sociale e culturale in città» («Corriere Romagna», 1 luglio 2016). Viene sottolineata l'importante valenza urbanistica dell'intervento, che toglierà dall'abbandono un luogo di interesse storicoarchitettonico situato in prossimità del centro storico e che potrà fare da traino alla rigenerazione urbana delle aree circostanti.

L'edizione 2017 del festival Ipercorpo ha preso forma dal lavoro di Città di Ebla e Spazi indecisi in stretta collaborazione con il Teatro Diego Fabbri di Forlì e con il progetto europeo Atrium (Architecture of Totalitarian Regimes): non a caso il tema è stato il patrimonio architettonico. Quest'anno, oltre a utilizzare gli spazi dell'ex deposito Atr, si sono mantenuti altri spazi (per esempio con il progetto ExAtr Lab - Case Acer, che ha aperto al pubblico alcune case popolari della zona attigua al deposito) e si è allungata la durata del festival, estendendo le iniziative per una ventina di giorni, dall' 8 al 28 maggio.

\section{Storia dello Stabilimento Sita / Deposito Atr}

Nel 1935 la Società italiana trasporti automobilistici, che apparteneva al gruppo Fiat, su espressa richiesta del capo del governo e dopo lunghe e incerte trattative con le autorità cittadine, consolidò la sua permanenza nella città di Forlì con la costruzione di una grande autorimessa. Essa avreb-

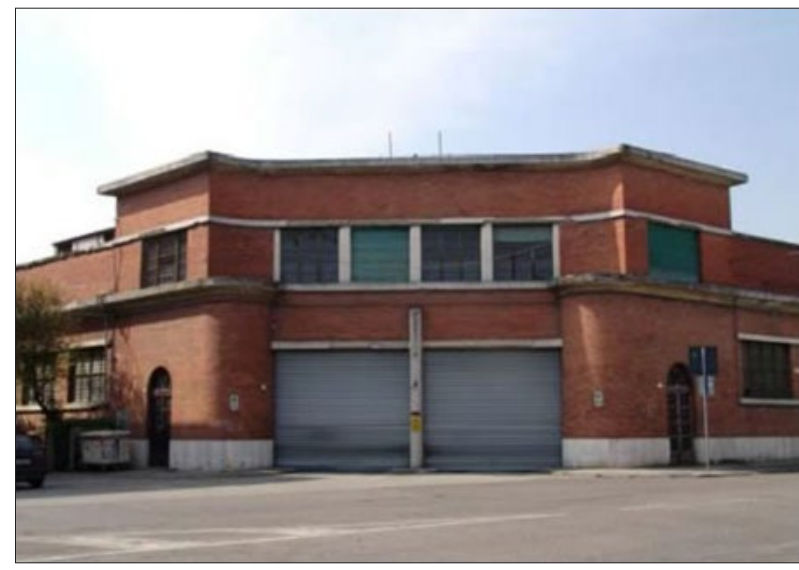

Fig. 3. L'ex deposito Atr oggi. be dovuto favorire i collegamenti interregionali fra Toscana e Marche. Il Direttore della filiale forlivese Luigi Bresciani affidò il progetto al geometra Alberto Flamigni [Tramonti 2005, 43]. La conformazione particolare del lotto e le sue esigenze funzionali suggerirono il posizionamento dell'ingresso principale carrabile sull'angolo accessibile direttamente dal grande piazzale che immetteva nei viali di circonvallazione. Il grande edificio, di natura prettamente industriale, fu realizzato al suo esterno in cotto con un basso basamento in travertino; la finestratura continua venne sot- 
tolineata da davanzali e architravi in cemento armato trattato a finto travertino, mentre l'ossatura della struttura fu realizzata prettamente in cemento armato. Il grande vano era caratterizzato dalla copertura a sheds, poggiando su grandi travi reticolari senza pilastri intermedi a sorreggerla [Mondini 2011]. Questo edificio, che porta in sé i tratti peculiari del periodo storico in cui è stato costruito, si inserì senza clamori all'interno del panorama di architetture razionaliste che negli anni del regime fascista contribuirono alla trasformazione architettonica della città di Forli.

L'ex deposito autocorriere Sita è stato inserito nel sistema dei principali interventi di recupero urbano del centro storico (ambito R.U. di iniziativa pubblica all'interno del P.C.S., Progetto Complementare 6), atto a rivitalizzare un settore del centro storico al momento notevolmente antipolare. Il riutilizzo con modificata destinazione di uso dell'edificio dismesso ex Sita potrà modificare l'assetto di questa parte del centro, valorizzandola e offrendo dunque alla città un'ulteriore opportunità di riqualificazione dell'area.

Per supportare le prossime iniziative di rigenerazione urbana che riguarderanno l'ex stabilimento Sita si è proceduto a un lavoro di ricerca sulle fonti riguardanti il progetto iniziale e la sua collocazione nell'urbanistica cittadina. Già pochi anni fa è stata pubblicata una tesi di laurea in architettura sul deposito, di Erika Mondini, che è stata presentata durante una passata edizione del festival Ipercorpo. Il lavoro, seppur poderoso e con valide indicazioni tecniche, propone degli interventi strutturali sul palazzo abbastanza consistenti: cosa che però si scontra con l'intento conservativo promosso da Città di Ebla e Spazi indecisi per il progetto Atr contemporaneo.

La prima tappa della ricerca si svolge all'Archivio di stato di Forlì, dove si trovano i documenti relativi alla costruzione dello stabilimento Sita. Qui sono reperibili alcune piante catastali (la più vecchia datata 9 marzo 1935); la richiesta del permesso per costruire del 22 luglio 1935 ed evasa il 5 agosto; una lettera del 7 agosto dove si richiede come variante una sopraelevazione di due metri nel corpo centrale; la concessione di inizio lavori datata 12 agosto 1935. Le date sono piuttosto ravvicinate, e coprono un arco temporale di circa 5 mesi: segno che i lavori procedettero celermente e senza particolari intoppi. I documenti, riguardanti la nuova sede all'angolo tra via Nazario Sauro e Ugo Bassi della Sita e con l'intestazione del podestà sono in perfetto stato di conservazione. Purtroppo non si può dire la stessa cosa delle mappe: molte sono rovinate ai bordi, comprensibilmente ingiallite dagli anni, e presentano un preoccupante sbiadimento degli inchiostri. Il secondo archivio visionato è il Fondo Piancastelli della Biblioteca di Forlì, dove sono state consultate le annate 1934, 1935 e 1936 del periodico fascista «Il 


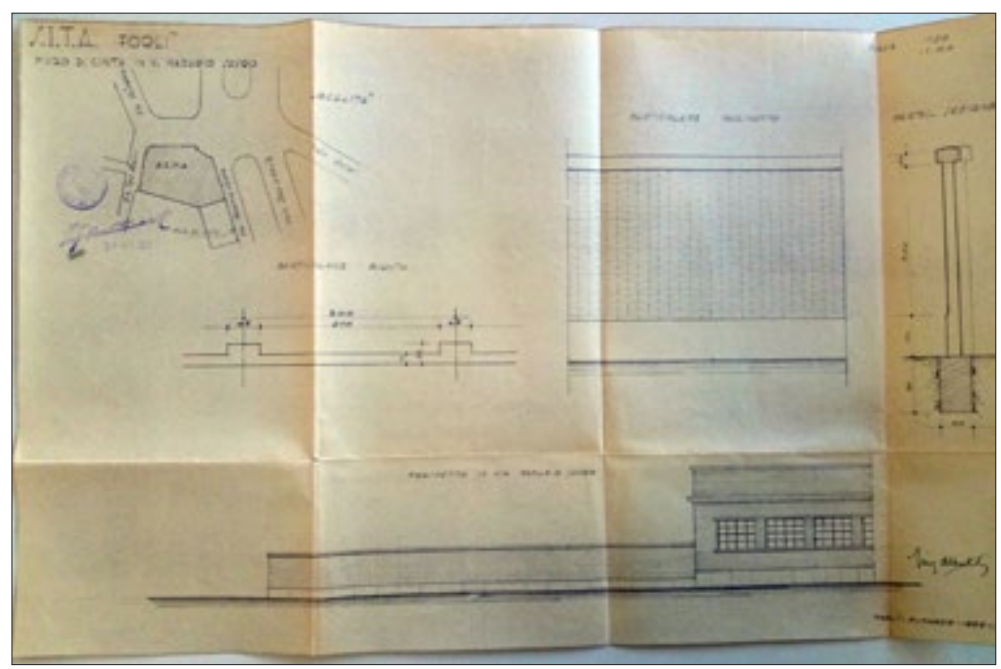

Fig. 4. Mappa del 9 marzo 1935, vidimata dall'ing. Montanari il 24 agosto (Archivio di stato di Forli).

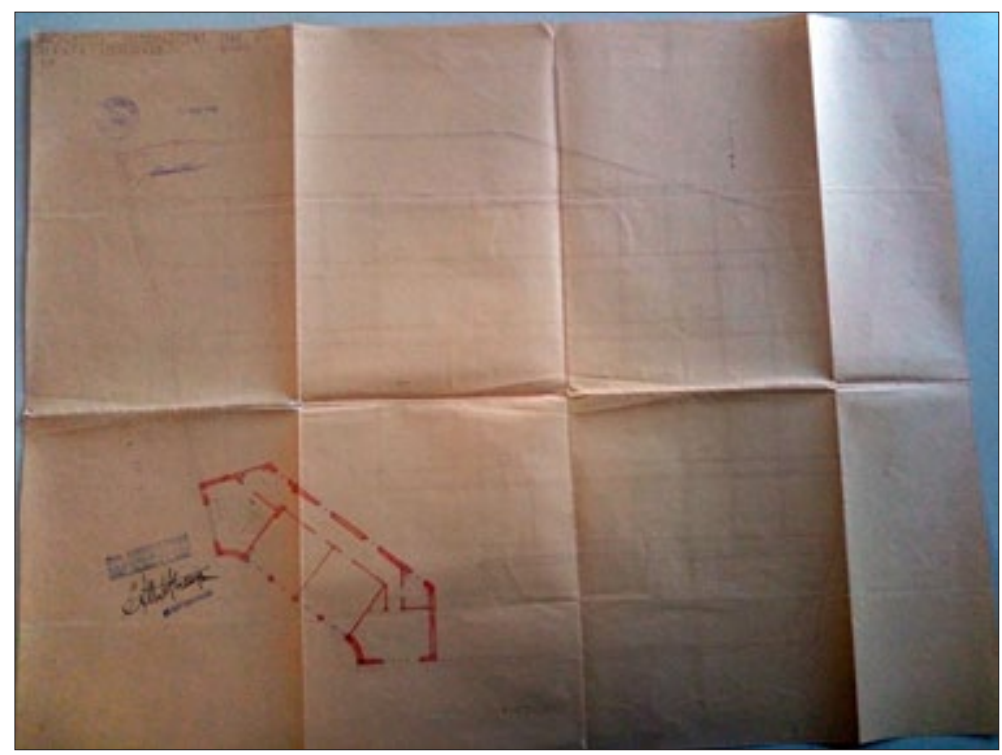

Fig. 5. Prospetto catastale del piano terra, con alcune notazioni quasi illeggibili (Archivio di Stato di Forli).

Popolo di Romagna». Qui non compaiono notizie sul nuovo stabilimento, ma il 16 agosto 1935 un'intera pagina è dedicata al nuovo piano regolatore di Forlì, il progetto Caesar XIII, di potenziamento delle infrastrutture del centro storico. Questo progetto comprende la costruzione dello stabilimento Sita. Sorprendentemente, nonostante l'importanza rivestita dall'edificio nel piano regolatore, non si è trovato invece materiale relativo alla sua inaugurazione.

Attraverso gli strumenti della public history è dunque possibile valorizzare il materiale archivistico relativo a edifici prima in disuso e poi restituiti con una nuova funzione alla città. La richiesta di storia, come possiamo quotidianamente toccare per mano nel nostro lavoro, è pressante, e viene dagli utenti più disparati. 


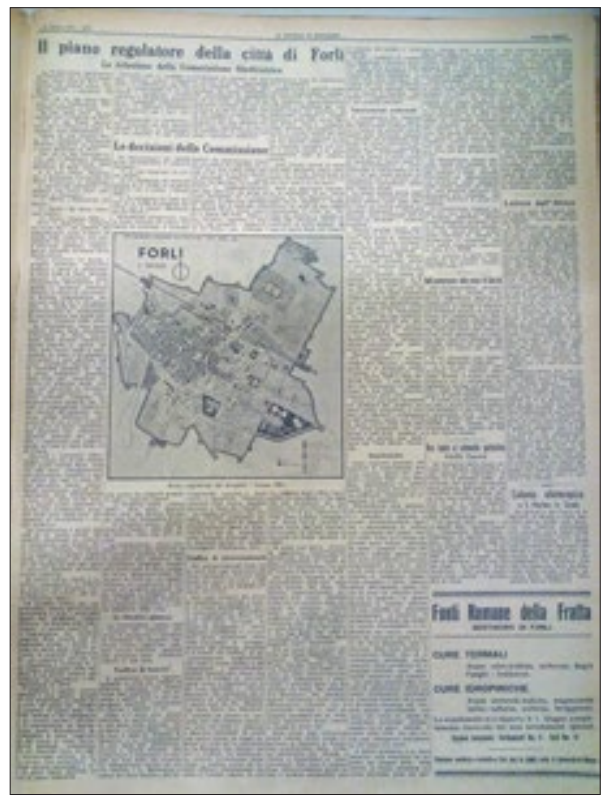

Fig. 6. Progetto Caesar XIII, «Il Popolo di Romagna», 16 agosto 1935 (Biblioteca di Forli, Fondo Piancastelli).

Intercettare l'utenza di un festival multidisciplinare come Ipercorpo rappresenta una sfida interessante. L'apporto dell'esperienza di Spazi indecisi attraverso la rigenerazione urbana leggera punterà l'attenzione su un luogo solitamente "indeciso" come il deposito delle corriere ormai da tempo abbandonato, che diventerà il contenitore di spettacoli di teatro, arte, musica.

\title{
Bibliografia
}

Mondini E. 2011, Deposito autocorriere S.I.T.A. a Forli 1935: Ipotesi di recupero e riabilitazione strutturale, Università di Bologna a.a. 2010-2011

Nicosanti E. 2015, Evento Ipercorpo: traiettorie di un progetto diventato festival, in Mei S. (ed.) 2015, La terza avanguardia: ortografie dell'ultima scena italiana, Lucca: La casa Usher

Tramonti U. 2005, Le radici del razionalismo in Romagna. Itinerari nel comprensorio forlivese, Forlì: Menabò

Troilo M. 2014, Vita sociale a Forli nel ventennio, consultabile sul sito di Atrium, http:// atrium.comune.forli.fc.it/introduzione-vita-sociale-a-forli-nel-ventennio/

\section{Risorse on line}

\author{
Progetto Atrium \\ http://atrium.comune.forli.fc.it/ \\ Associazione culturale Città di Ebla \\ http://www.cittadiebla.com/ \\ Spazi indecisi \\ http://www.spaziindecisi.it/
}

